\title{
Evidence for widespread subfunctionalization of splice forms in vertebrate genomes
}

\author{
Matthew J. Lambert, ${ }^{1}$ Wayne O. Cochran, ${ }^{2}$ Brandon M. Wilde, ${ }^{3}$ Kyle G. Olsen, ${ }^{3}$ \\ and Cynthia D. Cooper ${ }^{1,4}$ \\ ${ }^{7}$ School of Biological Sciences, Washington State University, Pullman, Washington 99164, USA; ${ }^{2}$ School of Engineering and \\ Computer Science, Washington State University Vancouver, Vancouver, Washington 98686, USA; ${ }^{3}$ College of Arts and Sciences, \\ Washington State University Vancouver, Vancouver, Washington 98686, USA; ${ }^{4}$ School of Molecular Biosciences, Washington State \\ University, Pullman, Washington 99164, USA
}

\begin{abstract}
Gene duplication and alternative splicing are important sources of proteomic diversity. Despite research indicating that gene duplication and alternative splicing are negatively correlated, the evolutionary relationship between the two remains unclear. One manner in which alternative splicing and gene duplication may be related is through the process of subfunctionalization, in which an alternatively spliced gene upon duplication divides distinct splice isoforms among the newly generated daughter genes, in this way reducing the number of alternatively spliced transcripts duplicate genes produce. Previously, it has been shown that splice form subfunctionalization will result in duplicate pairs with divergent exon structure when distinct isoforms become fixed in each paralog. However, the effects of exon structure divergence between paralogs have never before been studied on a genome-wide scale. Here, using genomic data from human, mouse, and zebrafish, we demonstrate that gene duplication followed by exon structure divergence between paralogs results in a significant reduction in levels of alternative splicing. In addition, by comparing the exon structure of zebrafish duplicates to the co-orthologous human gene, we have demonstrated that a considerable fraction of exon divergent duplicates maintain the structural signature of splice form subfunctionalization. Furthermore, we find that paralogs with divergent exon structure demonstrate reduced breadth of expression in a variety of tissues when compared to paralogs with identical exon structures and singletons. Taken together, our results are consistent with subfunctionalization partitioning alternatively spliced isoforms among duplicate genes and as such highlight the relationship between gene duplication and alternative splicing.
\end{abstract}

[Supplemental material is available for this article.]

Gene duplication has long been known as an important source of genetic novelty leading to functionally distinct proteins (Ohno 1970). Alternative splicing, through the differential retention of exonic and in some cases intronic material, likewise generates novel protein sequences (Black 2003). There are several types of alternative splicing events, including cassette exons, alternative $5^{\prime}$ and $3^{\prime}$ splice sites, mutually exclusive exons, and intron retention, with cassette exons being the most common in vertebrates. Interestingly, rates of alternative splicing correlate with organismal complexity, and it is alternative splicing that bridges the gap between the $\sim 22,000$ genes within the human genome and the more than 100,000 unique proteins that the human genome codes for (Keren et al. 2010). Given that both alternative splicing and gene duplication increase proteomic diversity, it is not surprising that several investigators have hypothesized that the two processes may be evolutionarily related. One manner in which alternative splicing and gene duplication may be related is through subfunctionalization, wherein an alternatively spliced gene upon duplication partitions distinct splice isoforms among the newly generated daughter genes (paralogs) (Hughes 1994; Force et al. 1999). In the context of subfunctionalization, gene duplication substitutes for alternative splicing, thus freeing duplicate genes from the constraints of maintaining multiple functions (Hughes 1994; Hahn 2009).

Corresponding author: cdcooper@vancouver.wsu.edu

Article published online before print. Article, supplemental material, and publication date are at http://www.genome.org/cgi/doi/10.1101/gr.184473.114.
Numerous individual gene case studies provide evidence that alternative splicing and gene duplication may be interchangeable. For example, Yu et al. (2003) demonstrate the subfunctionalization of the duplicated fugu (Takifugu rubripes) gene syn2 by showing that the two copies correspond to distinct isoforms of the alternatively spliced orthologous human gene. In a similar manner, Altschmied et al. (2002) and Hultman et al. (2007) demonstrate the subfunctionalization of the teleost genes mitf and kitl, respectively. Recently, Marshall et al. (2013) show that splice form subfunctionalization is not restricted to vertebrates. They report that in several fungal species two distinct proteins, SKI7 and HBS, are generated from a single alternatively spliced gene. In Saccharomyces cerevisiae, however, these isoforms were partitioned into distinct genes after gene duplication and subsequent subfunctionalization.

In addition to those listed above, several genome-wide studies have examined the relationship between alternative splicing and gene duplication. Kopelman et al. (2005) and Su et al. (2006) provide support for the relationship between gene duplication and alternative splicing by showing that as gene family size increases, levels of alternative splicing decrease. This negative correlation was presumed to be the product of subfunctionalization reducing

(C) 2015 Lambert et al. This article is distributed exclusively by Cold Spring Harbor Laboratory Press for the first six months after the full-issue publication date (see http://genome.cshlp.org/site/misc/terms.xhtml). After six months, it is available under a Creative Commons License (Attribution-NonCommercial 4.0 International), as described at http://creativecommons.org/licenses/by$\mathrm{nc} / 4.0 /$. 
alternative splicing levels in duplicate genes ( $\mathrm{Su}$ et al. 2006). Others, however, have questioned whether the inverse relationship between alternative splicing and gene duplication can be explained by subfunctionalization or whether the negative correlation between the two is even valid (Talavera et al. 2007; Jin et al. 2008; Chen et al. 2011; Roux and Robinson-Rechavi 2011; Grishkevich and Yanai 2014). Of note, Roux and Robinson-Rechavi (2011), using a more complete data set than either Kopelman et al. (2005) or Su et al. (2006), find that the largest gene families display the lowest levels of alternative splicing; however, singletons do not have the highest levels of alternative splicing. In fact, it would seem as though gene families of intermediate size undergo the most alternative splicing. This is difficult to reconcile with the hypothesis that gene duplication can substitute for alternative splicing, but suggests that less stringent purifying selection acting on newly formed duplicates may allow for the acquisition of novel splice forms (Roux and Robinson-Rechavi 2011). They also argue that the apparent reduction in alternative splicing found in the largest gene families is not due to gene duplication reducing levels of alternative splicing but is the product of a preduplication bias predisposing genes with initially low levels of alternative splicing to duplicate more frequently (Studer and Robinson-Rechavi 2009; Roux and Robinson-Rechavi 2011).

More recently, we have argued that the signature of splice form subfunctionalization for genes containing alternatively spliced cassette exons before duplication will be duplicate pairs with different numbers of exons. We have demonstrated that in zebrafish and fugu, duplicate pairs with different numbers of exons undergo less alternative splicing than singletons and duplicate pairs with identical numbers of exons. This reduction in alternative splicing is reflected in both the proportion of genes that undergo alternative splicing and mean number of transcripts per gene (Lambert et al. 2014).

The purpose of this study is to examine the relationship between alternative splicing and gene duplication in light of exon structure divergence after gene duplication on a genome-wide scale, in a variety of vertebrate species.

\section{Results}

\section{Exon divergent paralogs undergo less alternative splicing}

This study focuses on human (Homo sapiens), mouse (Mus musculus), and zebrafish (Danio rerio). These species were selected because they represent a wide range of vertebrates, and for each there exists a considerable amount of resources, including well-annotated genomes and an abundance of expression data. For each species we compiled a data set that consists of singletons and sibling paralogs (see Methods). Within the three species, between $4 \%$ and $26 \%$ of all genes are sibling paralogs that have diverged such that they now have different numbers of exons (Fig. 1A). Such change represents an extreme form of intron/exon structure divergence $(\mathrm{Xu}$ et al. 2012). We refer to these duplicate pairs with nonidentical numbers of exons as exon divergent paralogs. Those sibling paralogs that have retained the same number of exons are referred to as nondivergent paralogs. Previously, we have shown for a subset of genes in zebrafish that exon divergent duplicate pairs undergo significantly less alternative splicing compared with single-copy genes and nondivergent paralogs. Furthermore, by comparing the intron/exon structure of zebrafish paralogs to their alternatively spliced orthologs in human, we demonstrated splice form subfunctionalization (Fig. 1B) for a large portion of the exon diver- gent duplicate pairs (Lambert et al. 2014). Here, to test if the effects of splice form subfunctionalization are detectable at the level of entire genomes, we calculated the mean number of transcripts per gene for the singletons and exon divergent and nondivergent paralogs in each of the three species. In every case, the exon divergent paralogs have significantly fewer alternatively spliced transcripts per gene compared with both the singletons and nondivergent paralogs (Fig. 2A). In addition, we calculated the proportion of genes that are alternatively spliced, i.e., the fraction of genes with more than one transcript, for each group in each species. Again, we find that the exon divergent group consistently demonstrates a significant reduction in alternative splicing (Fig. $2 \mathrm{~B})$. Although the mean number of transcripts and proportion of genes with more than one transcript are both widely used to measure rates of alternative splicing, Su and Gu (2012) argue that a more accurate measure is the mean number of transcripts per gene, excluding genes with only a single transcript. Calculating levels of alternative splicing in this manner is more sensitive to rare alternative splicing events that likely remain undetected by current sequencing methods. Furthermore, by excluding genes with only a single transcript we eliminate the effects of duplication via retrotransposition, which creates nonalternatively spliced, intronless daughter genes regardless of the alternative splicing status of the parental gene (Kopelman et al. 2005). We performed this calculation and found that the exon divergent paralogs still demonstrate the lowest levels of alternative splicing in each species (Supplemental Fig. S1). It should be noted that we have included all transcripts, protein coding, and otherwise in these calculations. However, filtering the data set so that only protein coding transcripts are counted did not change the overall pattern. The exon divergent paralogs have the lowest levels of alternative splicing even when only protein coding transcripts are considered (Supplemental Fig. S2). Importantly, we find that the nondivergent paralogs consistently display the highest levels of alternative splicing, regardless of which measure of alternative splicing is used.

The above results suggest that gene duplication followed by exon structure divergence, but not gene duplication alone, may lead to a reduction in alternative splicing. However, there are several potentially confounding factors. First, it is known that gene families with many paralogs undergo very low rates of alternative splicing (Kopelman et al. 2005; Su et al. 2006; Jin et al. 2008; Chen et al. 2011; Roux and Robinson-Rechavi 2011). Therefore, if the exon divergent paralogs are enriched in genes that belong to these large gene families, the importance of exon structure divergence would necessarily be called into question as the association between exon structure divergence and lower rates of alternative splicing may be spurious, due to the influence of gene family size. To test for the effects of gene family size, we compared the number of paralogs per gene for the exon divergent and nondivergent groups. Singletons, by definition, have only one member in each family. Figure $3 \mathrm{~A}$ shows that the number of paralogs per gene is significantly different between the two groups in each species; however, it is the nondivergent paralogs, rather than the exon divergent paralogs, that have larger mean family sizes. These results indicate that family size is not responsible for the reduction in alternative splicing observed in the exon divergent group.

Another potentially confounding effect is based on the age of the duplication event that created the paralogs. Su et al. (2006) demonstrate that in humans there is a reduction in alternative splicing shortly after gene duplication. This reduction appears to be transient, as increasing time since duplication correlates with increasing levels of alternative splicing (Su et al. 2006; Roux and 
$\mathbf{A}$
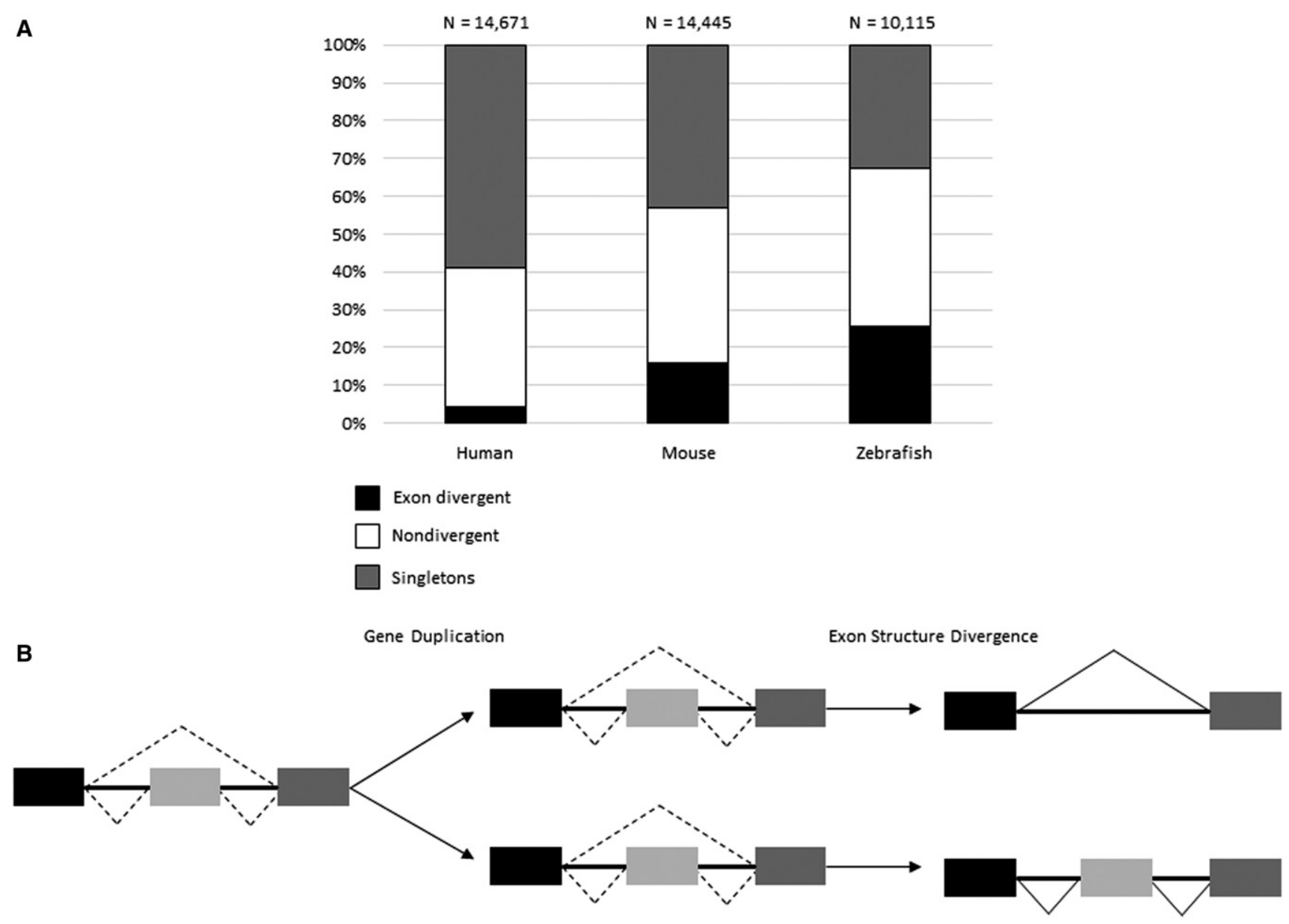

Figure 1. Gene duplication and exon structure divergence. (A) Stacked bar chart indicating the proportions of exon divergent paralogs, nondivergent paralogs, and singletons in each species. A total of 14,671,14,445, and 10,115 genes comprise the data sets for human, mouse, and zebrafish, respectively. (B) A model of exon structure divergence. An alternatively spliced gene prior to duplication codes for both a long and a short transcript. After duplication, the long transcript becomes fixed in one of the duplicates, while the short transcript becomes fixed in the other copy. In this way, each duplicate codes for only half of the ancestral alternative splicing repertoire. The dashed lines represent alternative splicing; solid lines, constitutive splicing events that have become fixed after duplication.

Robinson-Rechavi 2011). This means that if exon divergent paralogs are enriched in young duplicates, the influence of duplication age may make it appear as though exon structure divergence between paralogs results in lower levels of alternative splicing. Figure 3B plots the proportions of exon divergent and nondivergent paralogs created during a variety of evolutionary epochs as indicated by Ensembl Compara (Vilella et al. 2009). Within each plot, the proportions of divergent and nondivergent paralogs produced at each epoch are strikingly similar. Furthermore, in each species, except humans, there exists an enrichment of nondivergent paralogs in the most recent age categories. In humans, the proportion of nondivergent paralogs is slightly less than the proportion of exon divergent paralogs when considering the most recent duplication ages (Homo sapiens-Hominoidea); however, this excess is only marginally significant $\left(\chi^{2}=6.06, P=0.014\right)$. These results demonstrate that exon divergent paralogs are not enriched in young duplicates compared with nondivergent paralogs and that time since duplication is not a confounding factor.

Lastly, Grishkevich and Yanai (2014) use several genetic parameters, specifically gene length and expression level, to explain the inverse relationship between gene duplication and alternative splicing. They argue that shorter genes are more likely to duplicate while being less likely to undergo alternative splicing. Likewise, they argue that genes that are expressed at low levels demonstrate low levels of alternative splicing and high rates of duplication. These arguments provide a mechanism for the preduplication bias first proposed by Roux and Robinson-Rechavi (2011). That is to say, the apparent reduction in alternative splicing found in large gene families is not due to gene duplication, rather it is a consequence of short genes and lowly expressed genes being more likely to duplicate while being less likely to undergo alternative splicing. It is very unlikely that a preduplication bias is present in our data set because in each species only the exon divergent duplicates show any reduction in alternative splicing, while the nondivergent duplicates consistently demonstrate the highest levels of alternative splicing (Fig. 2). However, to demonstrate that gene length does not produce a confounding effect, we calculated the mean length of each category-exon divergent, nondivergent, and singletonin each species. Were gene length a confounding factor, we would expect the exon divergent paralogs to have the shortest lengths. Contrary to this expectation, we find that in each of the three species, exon divergent paralogs never have the shortest mean length

\section{Genome Research}

www.genome.org 

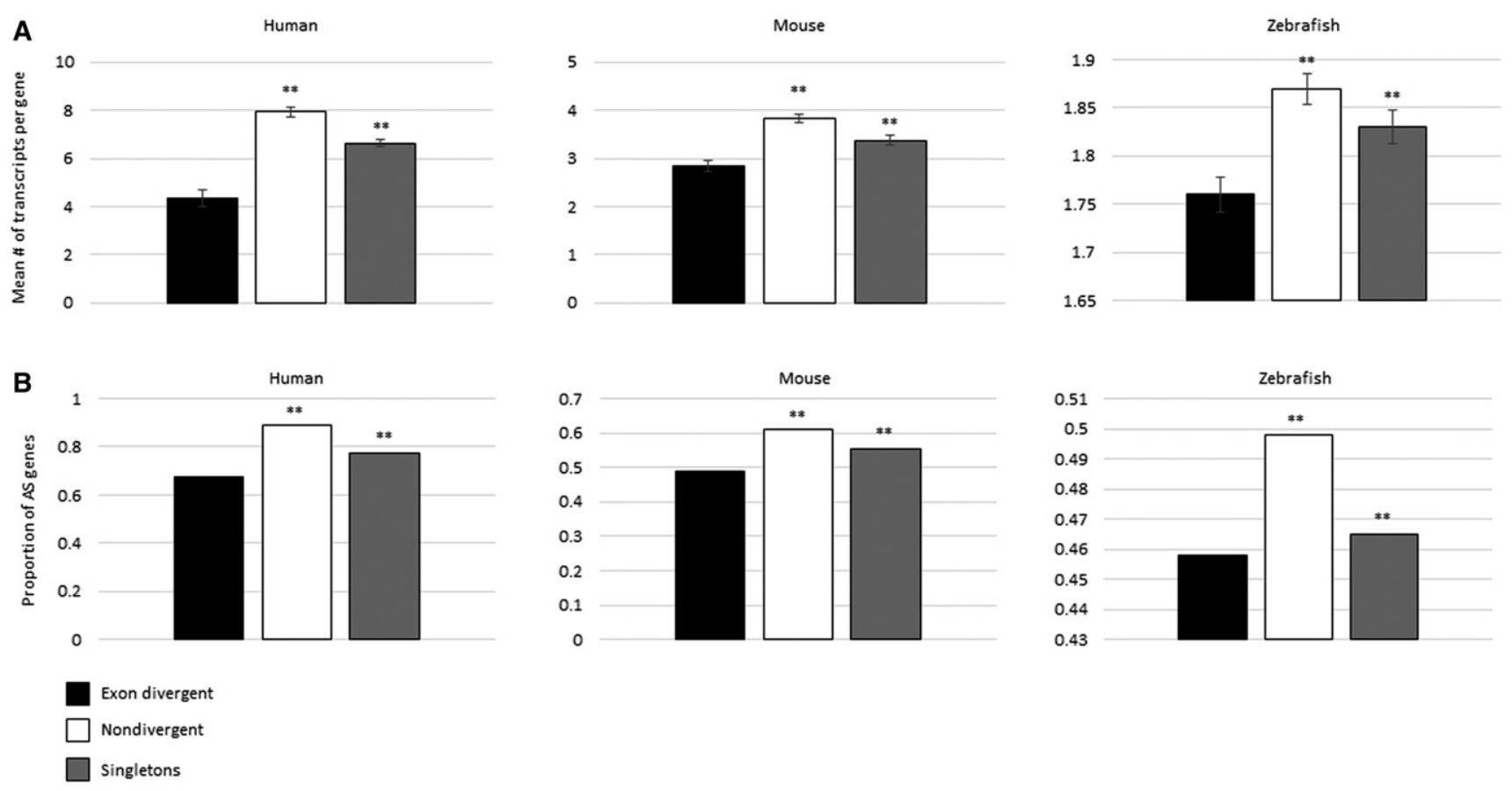

Figure 2. The relationship between exon structure divergence among paralogs and levels of alternative splicing. The exon divergent paralogs demonstrate significantly lower levels of alternative splicing compared to nondivergent paralogs and singletons as measured by mean number of transcripts $(A)$ and the proportion of genes that are alternatively spliced (AS) (B). Student's $t$-test was used to calculate the significance of the differences in mean number of transcripts. $\chi^{2}$ was used to calculate the significance of the differences in proportion of genes that are alternatively spliced. The asterisks indicate the significance of the difference as compared to exon divergent paralogs: $\left(^{* *}\right) P<0.01$. Error bars, SEM.

(Supplemental Fig. S3). Using UniGene expression data we also calculated the mean expression levels for each category in each species. We find that in accordance with Grishkevich and Yanai (2014), the exon divergent paralogs demonstrate low levels of expression; however, we believe this to be a consequence of subfunctionalization reducing the number of tissues in which the exon divergent paralogs are expressed (see below).

To further demonstrate that our results are not subject to these or any other potential preduplication biases, we queried the level of alternative splicing for all single-copy human genes that are orthologous to the genes that comprise the zebrafish data set. In this manner, the human orthologs serve as a preduplication outgroup, allowing us to approximate the ancestral state (Roux and Robinson-Rechavi 2011). These human orthologs were categorized based on the duplication state of the zebrafish genes, producing three groups: human orthologs of zebrafish singletons, zebrafish nondivergent paralogs, or zebrafish exon divergent paralogs. If any manner of bias were responsible for the reduction in alternative splicing witnessed by exon divergent paralogs, we would expect the human orthologs of the zebrafish exon divergent paralogs to have significantly lower levels of alternative splicing compared with the human orthologs of the zebrafish singletons or nondivergent paralogs. We find that the opposite is true: The human orthologs of the zebrafish exon divergent paralogs have the highest rates of alternative splicing (Fig. 4). We performed the same analysis with the mouse data set, again using orthologous human genes as a proxy for the ancestral state, and with human using mouse orthologs as the preduplication outgroup. In neither instance did we find a significant reduction in the levels of alternative splicing for the orthologs of the exon divergent paralogs (Fig. 4). These results indicate that the reduction in alternative splicing witnessed in the exon divergent paralogs is not the product of a preduplication bias.

\section{Exon divergent paralogs are expressed in fewer tissues}

The reduction in alternative splicing seen in the exon divergent paralogs suggests that splice form subfunctionalization may be driving down levels of alternative splicing. If this were true, we would expect the consequences of subfunctionalization to be manifest in other areas as well. For example, along with the partitioning of splice forms, expression profiles may also be divided among subfunctionalized duplicates. In this manner, subfunctionalization will result in duplicate genes with fewer transcripts and more restricted expression profiles. Indeed, shifts in expression after duplication have often been used as evidence for subfunctionalization (Blanc and Wolfe 2004; Casneuf et al. 2006; Duarte et al. 2006; Hellsten et al. 2007; Sémon and Wolfe 2008). To test if gene duplication followed by exon structure divergence results in the partitioning of expression domains, we analyzed the available UniGene (Pontius et al. 2003) EST expression profiles, which approximate expression patterns inferred from EST libraries. In line with our expectations, we find that in human and zebrafish the exon divergent paralogs are expressed in fewer tissue types than either singletons or nondivergent paralogs (Fig. 5). In mouse, the exon divergent paralogs are expressed in fewer tissues than singletons; however, there is no significant difference in the number of tissues between exon divergent and nondivergent paralogs. It may be that the reduced expression profiles evidenced in the exon divergent group are simply an effect of exon divergent paralogs possessing fewer transcripts per gene. To test this, we calculated the Pearson's correlation coefficient between the number of 
A

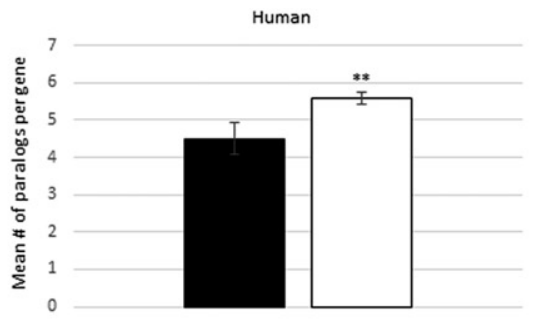

B

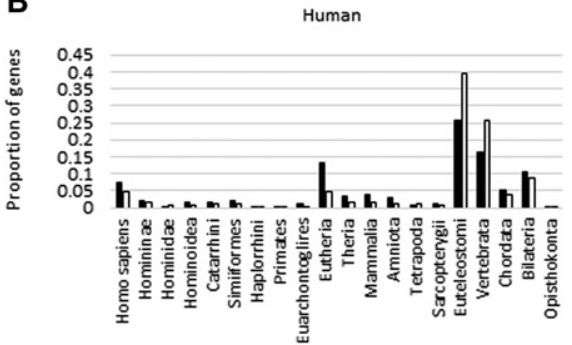

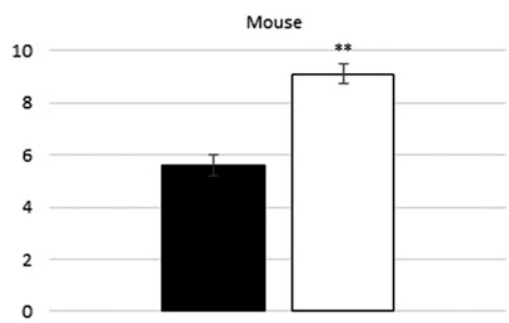

0

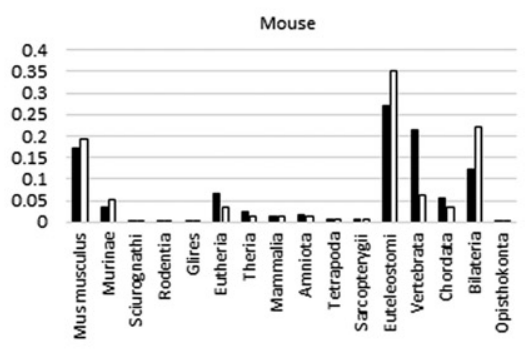

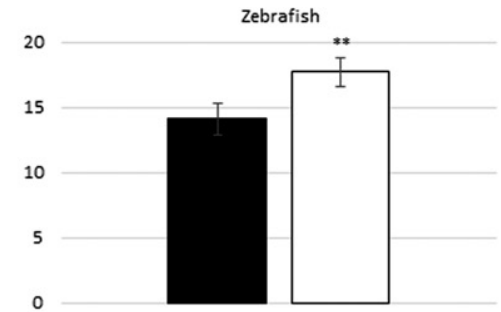

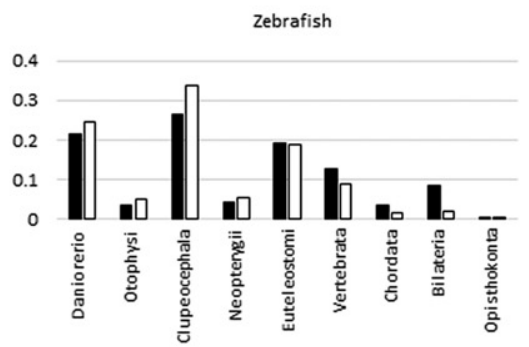

Exon divergent

Nondivergent

Figure 3. Family size and duplication age do not explain the reduction in alternative splicing. $(A)$ Histograms comparing gene family size between exon divergent and nondivergent paralogs. In each species the nondivergent paralogs have larger mean family sizes. Student's $t$-test was used to calculate the significance of the differences in mean number of paralogs. The asterisks indicate the significance of the difference as compared to exon divergent paralogs: $\left(^{* *}\right) P<0.01$. Error bars, SEM. (B) Histograms showing the proportion of all exon divergent paralogs (black) created at each evolutionary epoch in each species. These values are compared against the proportion of nondivergent paralogs (white) produced at each epoch within each species. (Note, for each species the black bars sum to one, as do the white bars.) Duplicate age increases along the $x$-axis (not to scale). These indicate that the exon divergent paralogs are not consistently enriched in young duplicates and that time since duplication is not a confounding effect.

tissues in which a gene is expressed and the number of transcripts a gene produces. We find that these parameters are correlated, however only very weakly (zebrafish $r=0.10$, mouse $r=0.16$, human $r=0.30$ ), suggesting that a reduction in alternative splicing does not, in and of itself, account for a restricted expression profile. In addition, to demonstrate that the restricted expression profiles found in the exon divergent paralogs are not simply a consequence of the selected data set, we performed the same analysis using the Electric Genetics/SANBI (Kelso et al. 2003) human gene expression data set. Again, we find that exon divergent paralogs are expressed in fewer tissue types (Supplemental Fig. S4).

\section{Structural evidence for splice form subfunctionalization}

The correspondence of exon structure between unique copies in a given sibling pair to distinct alternatively spliced transcripts from a preduplication outgroup gene serves as a signature of splice form subfunctionalization. To determine if the exon divergent duplicates maintain this signature of subfunctionalization, we compared the exon structure of a subset of zebrafish exon divergent duplicates to their co-orthologous gene in human, looking specifically for instances in which each duplicate in a given sibling pair shares a common exon structure with distinct alternatively spliced transcripts form the single human ortholog. Despite the fact that
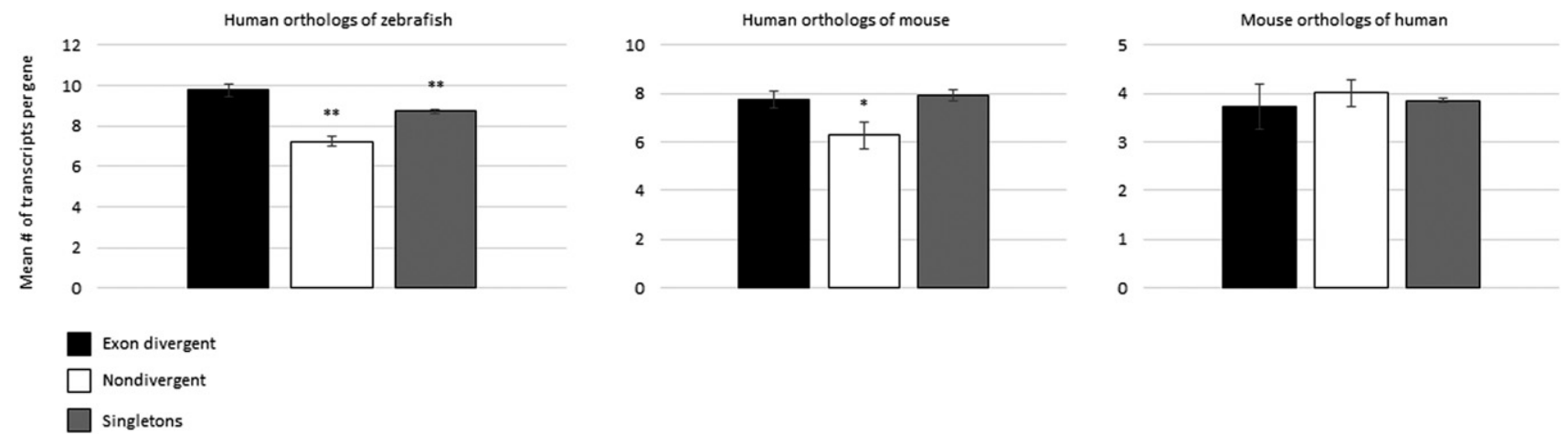

Figure 4. The potential for a preduplication bias. Histograms comparing the levels of alternative splicing in single-copy orthologs that serve as a proxy for the ancestral state. (Left) Human orthologs of zebrafish exon divergent paralogs (black) display higher levels of alternative splicing compared to the human orthologs of zebrafish nondivergent paralogs (white) and zebrafish singletons (gray). (Middle) Human orthologs of mouse exon divergent paralogs (black), nondivergent paralogs (white), and singletons (gray). (Right) Mouse orthologs of human exon divergent paralogs (black), nondivergent paralogs (white), and singletons (gray). The asterisks indicate the significance of the difference as compared to the singleton orthologs of the exon divergent paralogs: $(* *) P$ $<0.01\left(^{*}\right) P<0.05$. Error bars, SEM.

\section{Genome Research}

www.genome.org 

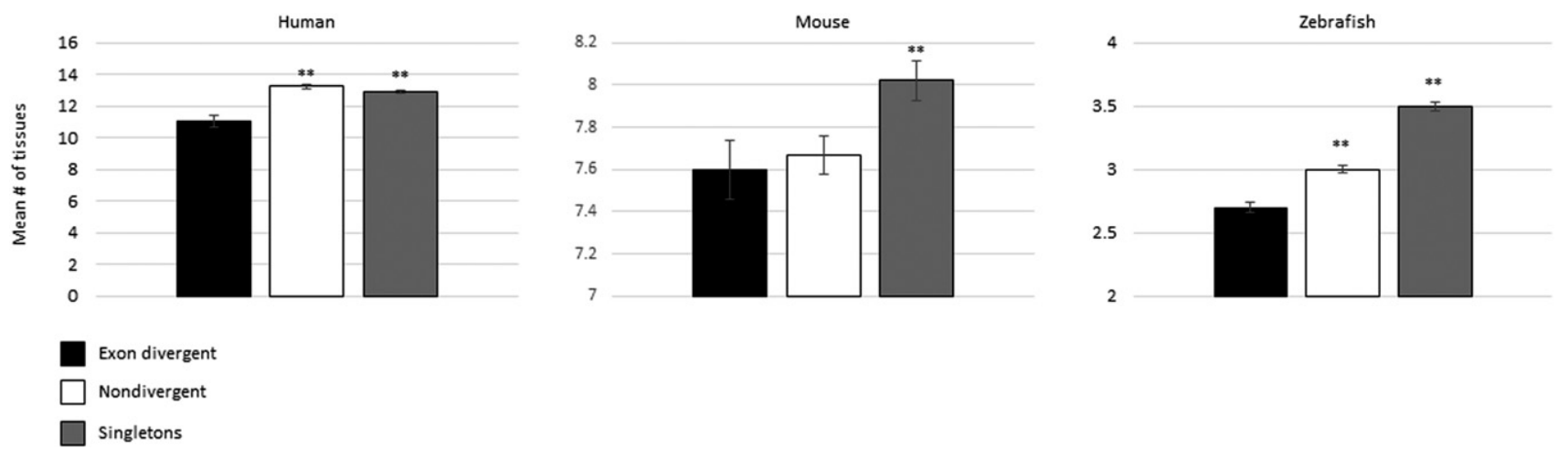

Figure 5. Exon divergent paralogs display restricted expression profiles. Exon divergent paralogs are expressed in fewer tissue types than nondivergent paralogs and singletons in each species. The $P$-values indicate the significance of the difference as compared to exon divergent paralogs. Student's $t$-test was used to calculate levels of significance. The asterisks indicate the significance of the difference as compared to exon divergent paralogs: $\left.{ }^{* *}\right) P<0.01$. Error bars, SEM.

the independent gain and loss of introns and exons along each lineage is expected to mask the signature of subfunctionalization, we find that $25 \%$ of those tested demonstrate a pattern that is indicative of splice form subfunctionalization (Supplemental Table 1). Figure 6 illustrates the splice form subfunctionalization of the gene cyclin M2 (cnnm2) in zebrafish. The human ortholog of cyclin M2, CNNM2, indicates that prior to duplication, the single copy gene coded for both a long and a short transcript. The long transcript contains eight exons and codes for a peptide that is 875 amino acids long. The short transcript contains seven exons and codes for an 853-amino-acid peptide; however, this transcript does not express the sixth exon found in the long transcript. In zebrafish, cnnm $2 a$ codes for a single transcript that contains eight exons, all of which correspond to the eight exons found in the long transcript of the human ortholog. The zebrafish paralog of cnnm $2 a$, cnnm $2 b$, shares a nearly identical exon structure with the short human transcript. The differential retention of the human cassette exon in the zebrafish duplicates suggests that the cnnm2 paralogs in zebrafish have been retained due to splice form subfunctionalization. Interestingly, tissue-specific expression patterns of zebrafish cnnm $2 a$ and cnnm $2 b$ (Arjona et al. 2013) largely mirror the expression patterns of the alternatively spliced transcripts of human CNNM2 (De Baaji et al. 2012).

\section{Discussion}

In 2005, Kopelman et al. (2005) demonstrated that a negative correlation between alternative splicing and gene duplication exists in the human genome. Later, Su et al. (2006) expanded upon this work and used the asymmetric evolution of alternative splicing between paralogs as evidence for subfunctionalization. Since then, a number of reports have been published that challenge whether the inverse correlation between alternative splicing and gene duplication is a consequence of subfunctionalization. For example, Roux and Robinson-
Rechavi (2011) invoke a preduplication bias and progressive acquisition of splice forms to explain the inverse relationship between alternative splicing and gene duplication. In a similar manner, Grishkevich and Yanai (2014) suggest that the reduction in alternative splicing seen in large gene families is explained by the propensity of short genes and lowly expressed genes, both of which are less likely to be alternatively spliced, to undergo duplication at higher rates.

Here, by considering exon divergent paralogs apart from other genes, we have focused on those duplicate pairs that are more likely to have been retained via subfunctionalization and as such have more power to detect the consequences of subfunctionalization. It should be noted that we do not argue that all duplicates with different numbers of exons are the product of subfunctionalization. Indeed, recent research suggests that exon structure divergence after duplication is much too common for subfunctionalization to be the single driver of divergence $\mathrm{Xu}$ et al. 2012; Wang et al. 2013). For example, processes such as intron/exon gain and loss and tandem exon duplication have the

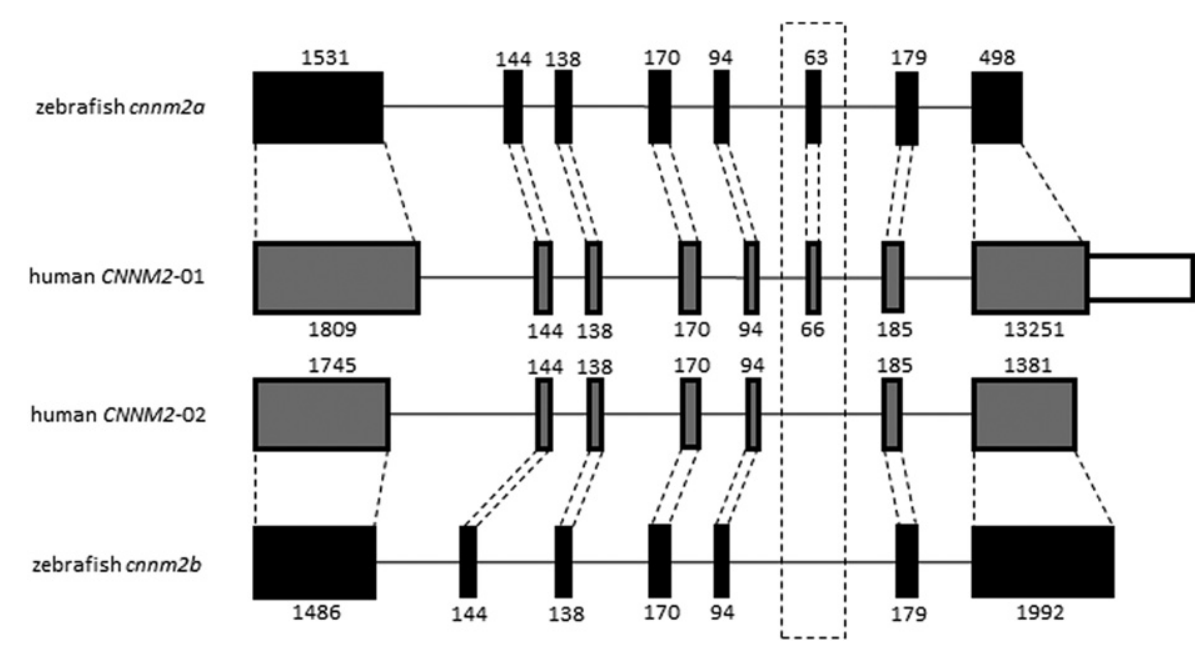

Figure 6. Structural evidence of splice form subfunctionalization. The zebrafish paralogs $c n n m 2 a$ and $c n n m 2 b$ correspond to distinct isoforms of the alternatively spliced orthologous human gene CNNM2. The dashed box highlights the alternatively spliced cassette exon from the human gene that has been subfunctionalized in the zebrafish paralogs. The dashed lines connect homologous exons. Exons lengths are indicated either below or above exons. Introns are not to scale. 
potential to cause exon structure divergence between paralogous genes but would not necessarily be involved in splice form subfunctionalization. Nevertheless, we argue that changes in intron/ exon structure after duplication should be accounted for, and in doing so we show that paralogs with different numbers of exons undergo the lowest rates of alternative splicing, as measured by mean number of transcripts per gene, as well as the proportion of genes with more than one transcript. We also show that exon divergent paralogs have restricted expression profiles, which is also consistent with subfunctionalization. Additionally, we demonstrate that the reduction in alternative splicing that accompanies exon structure divergence after gene duplication is free of all confounding factors that have previously been used to dismiss the relationship between alternative splicing and gene duplication.

Furthermore, we used the alternative splicing levels of singlecopy orthologs that diverged before duplication to approximate the ancestral preduplication state. In this way, we compared the number of isoforms between human genes that are orthologous to zebrafish singletons, zebrafish nondivergent paralogs, or zebrafish exon divergent paralogs. We find that the human orthologs of the zebrafish exon divergent paralogs display the highest rates of alternative splicing, indicating that the preduplication state may have been enriched in alternatively spliced isoforms. This is compelling evidence in support of our hypothesis that exon divergence subsequent to duplication substitutes for alternative splicing.

It should be noted that by studying paralogs with divergent exon numbers, we have focused on the subfunctionalization of genes that originally contained cassette exons. In truth, paralogs can have the same number of exons and still represent structural variants. However, this pattern indicates that the ancestral state (before duplication and subfunctionalization) included mutually exclusive exons or alternative $3^{\prime}$ or $5^{\prime}$ splice sites. We chose not to study these other types of alternative splicing because cassette exons represent the most common form of alternative splicing (Sorek et al. 2004; Pohl et al. 2013) and because detecting subfunctionalization of alternative $3^{\prime}$ and $5^{\prime}$ splice sites, as well as mutually exclusive exons, is computationally very difficult. By omitting other types of alternative splicing events, our estimates of the effects of subfunctionalization are likely conservative, meaning that gene duplication may reduce alternative splicing levels even more than reported here.

One point worth making is that the magnitude of the effect of subfunctionalization varies considerably between species. These differences are especially evident when comparing human and zebrafish. For example, in the human data set, alternative splicing levels are reduced nearly 50\% when comparing exon divergent and nondivergent paralogs. In zebrafish there is only a $6 \%$ reduction. This difference is likely the consequence of the relative contribution of gene duplication and alternative splicing to genome expansion in each lineage. Zebrafish have very high rates of gene duplication (Lu et al. 2012) and display very low levels of alternative splicing (Lu et al. 2010), while the inverse is true for humans (Shoja and Zhang 2006; Keren et al. 2010). Therefore, it is not surprising that the effects of subfunctionalization will be amplified in a genomic background replete with alternative splicing yet relatively free of duplicate genes, as is the case with the human genome. Another possibility is that differences in gene annotation quality among the three species may be responsible for the differences apparent in our data set. Ensembl's gene annotation pipeline relies heavily on the quality of the genome assembly, which can be assessed in several different ways, including scaffold and con- tig N50, as well as the number of ESTs and cDNAs aligned to the genome. The human genome has the highest quality assembly with substantially longer scaffold and contig N50 values, as well as many more aligned cDNAs and ESTs than either the mouse or zebrafish genomes. Considering that the human genome has the highest quality annotation, as well as the lowest proportion of exon divergent duplicates, it may be that some of the mouse and zebrafish exon divergent sibling pairs are the product of dubious annotation. However, the human genome demonstrates the largest effect of exon structure divergence between duplicates, meaning that we witness the greatest reduction in rates of alternative splicing in the human exon divergent duplicates. This suggests that any annotation artifacts present in the mouse and zebrafish data set may obscure rather than enhance the effects of splice form subfunctionalization and demonstrates that the reported results of exon structure divergence between paralogs are not the product of poor annotation.

In conclusion, we find that exon divergent duplicate genes undergo the lowest levels of alternative splicing and have restricted expression profiles, which is consistent with subfunctionalization partitioning distinct isoforms among duplicates. Considering that duplicate gene retention via subfunctionalization is thought to be relatively rare (Sémon and Wolfe), it is not surprising that the relationship between gene duplication and alternative splicing was not readily detected in previous genome-wide studies that treated all duplicates as equals.

\section{Methods}

\section{Sequence analysis}

All sequences were obtained from the Ensembl database release 75 (Cunningham et al. 2015). Ensembl Compara was used to group all genes in each species as either paralogs or singletons. Paralogs in each species were then subject to a species-specific reciprocal BLASTP search in an attempt to locate the most closely related sibling paralogs. BLAST $e$-values indicate that in each species most sibling paralogs are closely related. For example, $65 \%$ of the human, $66 \%$ of the mouse, and $74 \%$ of zebrafish BLAST $e$-values for sibling paralogs were below E-100, while 86\%, 85\%, and $90 \%$ were below E-50 in human, mouse, and zebrafish, respectively. Ensembl BioMart (Smedley et al. 2009) was used to count the total number of transcripts, the number of protein coding transcripts, and the number of exons for each gene. For independent estimates of the number of exons per gene we used the UCSC Genome Browser (Kent et al. 2002) to query RefSeq. In addition, we used ExoLocator (Khoo et al. 2014) to verify exon counts for a subset of genes in each species. Paralogs were classified as exon divergent if none of the transcripts contained equal numbers of exons. Information regarding duplication age, gene family size, and length was obtained directly from Ensembl.

We used Ensembl Compara to identify orthologous preduplication outgroup genes in mouse, with which we tested for a preduplication bias in humans. For the exon divergent and nondivergent paralog groups, we considered only genes that had duplicated since the divergence of human and mouse. We used a BLASTP mutual best hit approach to verify co-ortholog assignments. In this way, we only considered human duplicate pairs that identified a common paralog in mouse by both tree reconciliation and mutual best hit. This same analysis was performed for zebrafish using human single-copy orthologs to estimate the ancestral state and for mouse, again using human orthologs to estimate the ancestral state. 
We employed a two-step process to test for structural evidence of splice form subfunctionalization. First, exon sequences of protein coding transcripts for exon divergent zebrafish duplicates, as well as the single human co-ortholog, were aligned using tBLASTx. We used an expected value of $10^{-8}$ as a cutoff for determining homologous exons between species, although most expect values were much lower. We then used BLAT and the UCSC Genome Browser to verify these alignments against both the human genome and the zebrafish genome. This analysis was performed on the 100 zebrafish exon divergent sibling pairs with the most closely related human co-ortholog.

\section{Gene expression}

UniGene EST expression profiles were downloaded from UniGene (ftp://ftp.ncbi.nih.gov/repository/UniGene/; April 18, 2014). To estimate expression levels, we queried the profile for each species. In humans, there are 45 potential tissues that serve as sources for cDNA libraries. In mouse and zebrafish there are 43 and 8 potential tissue types, respectively. Following the method of Chain et al. (2011), we calculated three summary statistics regarding gene expression: total expression levels $(T)$, expression intensity $(I)$, and evenness $(E)$. The value $T$ measures transcripts per million summed across all tissue restricted libraries in which a gene is expressed $\left(T=\Sigma L_{\mathrm{i}}\right)$. It should be noted that genes that are highly expressed in one or a few tissues will have comparable $T$-values to those genes that are expressed in many tissues at lower levels. To distinguish between the two, we use the value $I$, which is calculated as a weighted average of expression levels: $I=\Sigma L_{\mathrm{i}}^{2} / \Sigma L_{\mathrm{i}}$. The value $T / I=E$ indicates the breadth of a gene's expression profile and is used here as a measure of the effective number of tissues in which a gene is expressed.

\section{Acknowledgments}

We thank Dr. Ruth Phillips and Kersten Peterson for their thoughtful comments on the manuscript. M.J.L. is supported by the Washington State University graduate student professional development award and the Washington State University College of Arts and Sciences Research and Creative Activity Enhancement Fund awarded to C.D.C.

Author contributions: M.J.L. conceived of the study and wrote the manuscript. M.J.L., W.O.C., K.G.O., and B.M.W. performed the experiments. C.D.C. edited the manuscript.

\section{References}

Altschmied J, Delfgaauw J, Wilde B, Duschl J, Bouneau L, Volff JN, Schartl M. 2002. Subfunctionalization of duplicate mitf genes associated with differential degeneration of alternative exons in fish. Genetics 161: 259-267.

Arjona FJ, Chen YX, Flik G, Bindels RJ, Hoenderop JG. 2013. Tissue-specific expression and in vivo regulation of zebrafish orthologues of mammalian genes related to symptomatic hypomagnesemia. Pflugers Arch 465: 1409-1421.

Black DL. 2003. Mechanisms of alternative pre-messenger RNA splicing. Annu Rev Biochem 72: 291-336.

Blanc G, Wolfe KH. 2004. Functional divergence of duplicated genes formed by polyploidy during Arabidopsis evolution. Plant Cell 16: 1679-1691.

Casneuf T, De Bodt S, Raes J, Maere S, Van de Peer Y. 2006. Nonrandom divergence of gene expression following gene and genome duplications in the flowering plant Arabidopsis thaliana. Genome Biol 7: R13.

Chain FJ, Dushoff J, Evans BJ. 2011. The odds of duplicate gene persistence after polyploidization. BMC Genomics 12: 599.

Chen TW, Wu TH, Ng WV, Lin WC. 2011. Interrogation of alternative splicing events in duplicated genes during evolution. BMC Genomics. 12 (Suppl 3): S16.
Cunningham F, Ridwan Amode M, Barrell D, Beal K, Billis K, Brent S, Carvalho-Silva D, Clapham P, Coates G, Fitzgerald S, et al. 2015. Ensembl 2015. Nucleic Acids Res 43: D662-D669.

De Baaji JH, Stuiver M, Meij IC, Lainez S, Kopplin K, Venselaar H, Muller D, Bindels RJ, Hoenderop JG. 2012. Membrane topology and intracellular processing of cyclin M2 (CNNM2). J Biol Chem 287: 1364413655.

Duarte JM, Cui L, Wall PK, Zhang Q, Zhang X, Leebens-Mack J, Ma H, Altman N, dePamphilis CW. 2006. Expression pattern shifts following duplication indicative of subfunctionalization and neofunctionalization in regulatory genes of Arabidopsis. Mol Biol Evol 23: $469-478$.

Force A, Lynch M, Pickett FB, Armores A, Yan Y, Postlewhait J. 1999. Preservation of duplicate genes by complementary, degenerative mutations. Genetics 151: $1531-1545$.

Grishkevich V, Yanai I. 2014. Gene length and expression level shape genomic novelties. Genome Res 24: 1497-1503.

Hahn MW. 2009. Distinguishing among evolutionary models for the maintenance of gene duplicates. J Hered 100: 605-617.

Hellsten U, Khokha MK, Grammer TC, Harland RM, Richardson P, Rokhsar DS. 2007. Accelerated gene evolution and subfunctionalization in the pseudotetraploid frog Xenopus laevis. BMC Biol 5: 31.

Hughes AL. 1994. The evolution of functionally novel proteins after gene duplication. Proc Biol Sci 256: 119-124.

Hultman KA, Bahary N, Zon LI, Johnson SL. 2007. Gene duplication of the zebrafish kit ligand and the partitioning of melanocyte development functions to kit ligand a. PLoS Genet 3: e17.

Jin L, Kryukov K, Clemente JC, Komiyama T, Suzuki Y, Imanishi T, Ikeo K, Gojobori T. 2008. The evolutionary relationship between gene duplication and alternative splicing. Gene 427: 19-31.

Kelso J, Visagie J, Theiler G, Christoffels A, Bardien S, Smedley D, Otgaar D, Greyling G, Jongeneel CV, McCarthy MI, et al. 2003. eVOC: a controlled vocabulary for unifying gene expression data. Genome Res 13: 12221230.

Kent WJ, Sugnet CW, Furey TS, Roskin KM, Pringle TH, Zahler AM, Haussler D. 2002. The Human Genome Browser at UCSC. Genome Res 12: 996-1006.

Keren H, Lev-Maor G, Ast G. 2010. Alternative splicing and evolution: diversification, exon definition and function. Nat Rev Genet 11: 345355.

Khoo AA, Ogrizek-Thomas M, Bulovic A, Korpar M, Gurler E, Slijepcevic I, Sikic M, Mihalek I. 2014. ExoLocator: an online view into genetic makeup of vertebrate proteins. Nucleic Acids Res 42: D879-D881.

Kopelman NM, Lancet D, Yanai I. 2005. Alternative splicing and gene duplication are inversely correlated evolutionary mechanisms. Nat Genet 37: $588-589$.

Lambert MJ, Olsen KG, Cooper CD. 2014. Gene duplication followed by exon structure divergence substitutes for alternative splicing in zebrafish. Gene 546: 271-276.

Lu J, Peatman E, Wang WQ, Yang Q, Abernathy J, Wang SL, Kucuktas H, Liu ZJ. 2010. Alternative splicing in teleost fish genomes: same-species and cross-species analysis and comparisons. Mol Genet Genomics 283: 531-539.

Lu J, Peatman E, Tang H, Lewis J, Liu Z. 2012. Profiling of gene duplication patterns of sequenced teleost genomes: evidence for rapid lineage-specific genome expansion mediated by recent tandem duplications. BMC Genomics 13: 246-257.

Marshall AN, Montealegre MC, Jiménez-López C, Lorenz MC, van Hoof A. 2013. Alternative splicing and subfunctionalization generates functional diversity in fungal proteomes. PLoS Genet 9: e1003376.

Ohno S. 1970. Evolution by gene duplication. Springer-Verlag, Berlin, Germany.

Pohl M, Bortfeldt RH, Grutzmann K, Schuster S. 2013. Alternative splicing of mutually exclusive exons: a review. Biosystems 114: 31-38.

Pontius JU, Wagner L, Schuler GD. 2003. UniGene: a unified view of the transcriptome. In The NCBI Handbook. National Center for Biotechnology Information, Bethesda, MD

Roux J, Robinson-Rechavi M. 2011. Age-dependent gain of alternative splice forms and biased duplication explain the relation between splicing and duplication. Genome Res 21: 357-363.

Sémon M, Wolfe KH. 2008. Preferential subfunctionalization of slow-evolving genes after allopolyploidization in Xenopus laevis. Proc Natl Acad Sci 105: 8333-8338.

Shoja V, Zhang L. 2006. A roadmap of tandemly arrayed genes in the genomes of human, mouse, and rat. Mol Biol Evol 11: 2134-2141.

Smedley D, Haider S, Ballester B, Holland R, London D, Thorisson G, Kasprzyk A. 2009. BioMart-biological queries made easy. BMC Genomics 10: 22

Sorek R, Shemesh R, Cohen Y, Basechess O, Ast G, Shamir R. 2004. A nonEST based method for exon-skipping prediction. Genome Res 14: 1617-1623. 


\section{Lambert et al.}

Studer RA, Robinson-Rechavi M. 2009. How confident can we be that orthologs are similar, but paralogs differ? Trends Genet 25: 210-216.

$\mathrm{Su} \mathrm{Z}$, Gu X. 2012. Revisit on the evolutionary relationship between alternative splicing and gene duplication. Gene 504: 102-106.

Su Z, Wang J, Yu J, Huang X, Gu X. 2006. Evolution of alternative splicing after gene duplication. Genome Res 16: 182-189.

Talavera D, Vogel C, Orozco M, Teichmann SA, de la Cruz X. 2007. The (in) dependence of alternative splicing and gene duplication. PLoS Comput Biol 3: e33.

Vilella AJ, Severin J, Ureta-Vidal A, Heng L, Durbin R, Birney E. 2009. EnsemblCompara GeneTrees: complete, duplication-aware phylogenetic trees in vertebrates. Genome Res 19: 327-335.
Wang Y, Tan X, Paterson AH. 2013. Different patterns of gene structure divergent following gene duplication in Arabidopsis. BMC Genomics 14: 652 .

Xu G, Guo C, Shan H, Kong H. 2012. Divergence of duplicate genes in exonintron structure. Proc Natl Acad Sci 109: 1187-1192.

Yu WP, Brenner S, Venkatesh B. 2003. Duplication, degeneration and subfunctionalization of the nested synapsin-Timp genes in Fugu. Trends Genet 19: 180-183.

Received September 15, 2014; accepted in revised form March 16, 2015.

\section{Genome Research}




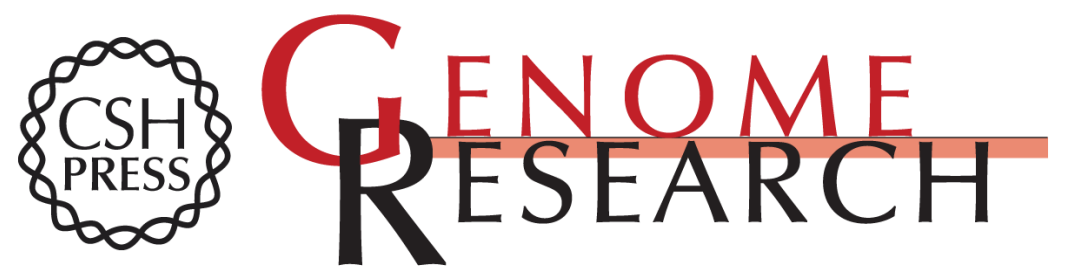

\section{Evidence for widespread subfunctionalization of splice forms in vertebrate genomes}

Matthew J. Lambert, Wayne O. Cochran, Brandon M. Wilde, et al.

Genome Res. 2015 25: 624-632 originally published online March 19, 2015

Access the most recent version at doi:10.1101/gr.184473.114

Supplemental Material

References

Creative

Commons

License

Email Alerting

Service
http://genome.cshlp.org/content/suppl/2015/03/19/gr.184473.114.DC1

This article cites 40 articles, 13 of which can be accessed free at: http://genome.cshlp.org/content/25/5/624.full.html\#ref-list-1

This article is distributed exclusively by Cold Spring Harbor Laboratory Press for the first six months after the full-issue publication date (see

$\mathrm{http}: / /$ genome.cshlp.org/site/misc/terms.xhtml). After six months, it is available under a Creative Commons License (Attribution-NonCommercial 4.0 International), as described at http://creativecommons.org/licenses/by-nc/4.0/.

Receive free email alerts when new articles cite this article - sign up in the box at the top right corner of the article or click here.

\section{Affordable, Accurate Sequencing.}

To subscribe to Genome Research go to:

https://genome.cshlp.org/subscriptions 